\title{
Thirty-three nucleotides of 5 ' flanking sequence including the 'TATA' box are necessary and sufficient for efficient U2 snRNA transcription in Schizosaccharo- myces pombe
}

\author{
T. Dandekar and D. Tollervey* \\ European Molecular Biology Laboratory, Postfach \\ 10.22.09, D-6900 Heidelberg, Germany.
}

\section{Summary}

We have sequenced the $5^{\prime}$ flanking region of the U2 gene and compared this with the $5^{\prime}$ flanking sequences of other snRNA genes from Schizosaccharomyces pombe. This revealed no regions of clear homology 5 ' to a region surrounding the 'TATA' box at $\mathbf{- 3 2}$ to -29 . Deletion analysis shows that a 5 ' flanking region extending to only -33 is sufficient for accurate and efficient transcription of U2 in Schizosaccharomyces pombe.

\section{Introduction}

The small nuclear RNAs (snRNAs), a group of stable low molecular-weight RNAs, are found in the nuclei of all eukaryotes examined to date. The most-studied snRNAs - U1, U2, U4, U5 and U6 - are all required for pre-messenger RNA splicing in vitro (reviewed by Maniatis and Reed, 1987) and in vivo (reviewed by Guthrie, 1988; Woolford, 1989). In recent years the transcription signals of genes encoding snRNAs have attracted considerable interest. Sequence comparisons and functional analyses show that snRNA genes of higher eukaryotes lack the 'TATA' sequences present upstream of most genes transcribed by RNA polymerase II, and instead have two important regions designated the proximal sequence element (PSE) and the distal sequence element (DSE). It is likely that the PSE and DSE play rples related to the TATA box and enhancer elements, respectively, of mRNA coding genes (Tebb and Mattaj, 1988). In contrast, snRNA genes in the budding yeast Saccharomyces cerevisiae have TATA sequences located between -80 and -100 (see Bally et al., 1988, for a compilation of such sequences), although results of their functional analysis have not been reported. In the fission yeast Schizosac-

Received 15 January, 1991; revised 19 March, 1991. *For correspondence. Tel. (6221) 387264; Fax (6221) 387306; E-mail Dandekar@ EMBL.Bitnet and Toliervey@EMBL.Bitnet. charomyces pombe, the polymerase II-transcribed snRNA genes (U1-U5) also contain TATA sequences (Brennwald et al., 1988; Dandekar et al., 1989; Porter et al., 1990). As is the case with mRNA coding genes (Russell, 1983), the TATA sequences in S. pombe snRNA genes lie closer to the transcription initiation site than do those of $S$. cerevisiae. Here we report the first functional analysis of the transcription signals in a S. pombe snRNA gene.

\section{Results and Discussion}

The cloning of the snu2 gene has previously been reported (Dandekar and Tollervey, 1989), and the sequence of the coding region together with the $5^{\prime}$ and $3^{\prime}$ flanking sequences is shown in Fig. 1. The sequence of the U2 RNA has been reported (Brennwald et al., 1988); the sequence shown in Fig. 1 differs in three positions from that previously reported (see the legend to Fig. 1).

Computer searches revealed no sequences in snu2 with clear homology to consensus sequences for the DSE (TATGCAAAY; Carbon et al., 1987) or PSE (TC(A/C)CTCTCCYYARG; Carbon et al., 1987) of vertebrate snRNA genes. In an effort to identify other transcription signals, the 5 flanking sequence of snu2 was aligned against the 5 flanking regions of snu1, snu3 and snu4. For U1 and U2, 5 ' flanking sequences extending to -386 were compared. The complete snu1 sequence determined, including the newly sequenced upstream region (an additional 147 upstream nucleotides), has been deposited in the EMBL/GenBank/DDBJ Nucleotide Sequence Data Libraries under the accession number $X 55773$. No differences were detected within the region previously sequenced (Porter et al., 1990). In the case of snu4, a region extending to position -230 is sufficient for efficient expression of the gene (Dandekar et al., 1989) and was used for comparison. For snu3 the published sequence extends to -315 (Porter et al., 1988), and this region was used for comparison. A region of homology was detected between the first two sequences available, i.e. snu2 and snu4. In the snu2 sequence this region lies at positions -71 to -56 . However, homologous sequences are not found in other snRNA genes, and deletion of this sequence between 

$-220$
$-210$
$-200$
$-190$
$-180$

snu2 TATATCATAT TGTTTTAAAA AATGTATGAA AATTTTTAGT GTAAAAAAAA
$-170$
$-160$
$-150$
$-140$
$-130$

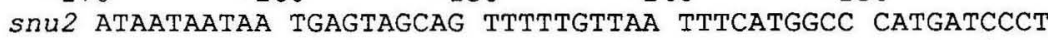
$-120$
$-110$
$-100$
$-90$
$-80$

snu2 TCTgGTAACA AGTTTGTCCA AGgCCTCtgA CCACAAAATT GGGACTGCGA

$-70$

$-60$

$-50$

$-40$

$-30$

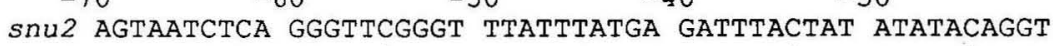
I U $2 \Delta 3$ $\mathrm{U} 2 \Delta 4 \quad \mathrm{U} 2 \Delta 5$ $-20$ $-10$ $+1$ 10

20 30 snu2 ACTCGTGCAT TTGgTTCGgT ATTCTCTCTT TGCCTTTTGg CTTAGATCAA U2

Gppp auUCuCucuU ugcCUUUUgg cuUAgaucaA

40

50

60

70

80

snu2 GTgtAgtATC TGTTCTTTTC AgtTtAatCG CTGAAATCAC CTCACTGAgG U2 GUGUAGUAUC UGUUCUUUUC AGUUUAAUCG CUGAAAUCAC CUCACUGAGg C in $\mathrm{M} 2$

$90 \quad 100 \quad 110 \quad 120 \quad 130$ snu2 TGTTTCCGAT TAATCTTGTT TTTGGTTTGA GTTGGAAAGC CTCTGGCTTG U2 UGUUUCCGAU UAAUCUUGUU UUUGGUUUGA GUUGGAAAGC CUCUGGCUUG
140
150
160
170
180

snu2 CTATGCTTTC CGACACTGGT GTTCTTGCTA TTGCACTACT GGCAAGCGAC U2 CUAUGCUUUC CGACACUGGU GUUCUUGCUA UUGCACUACU GGCAAGCGAC

\section{7}

$+10$

$+10+20$

$+30$

$+40$

snu2 GCCGAAT CTTCTTCTGT TGACAGATTT GTGACGCATG AATTTGACAA U2 GCCGAAUOH$$
-30
$$

snu1: CGTCGTTACTATAAATATGGGTGCTTACACTTACGGTTCTTT:^^^^^^^^^

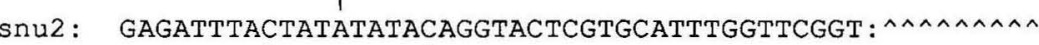
$\begin{array}{lc}\text { U } 2 \Delta 4 & \text { U } 2 \Delta 5\end{array}$$$
-30
$$$$
1
$$

snu3: GTTATT'TACTATATATACGGGTCATTATCCTACGAACAAAACACT: ^^^^^^$$
-30
$$

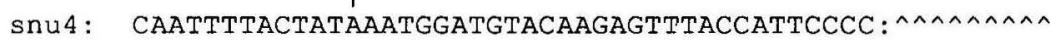

Fig. 1. Sequence of snu2. The coding sequence of snu2 is shown together with the $5^{\prime}$ flanking sequence extending to -220 , and the 3 ' flanking sequence extending to +64 . The end-points of deletions $\Delta 1-\Delta 5$ are shown. A vertical line denotes the $3^{\prime} \mathrm{C}$ of the $E c 0 \mathrm{RI}$ site at the $5^{\prime}$ end of each PCR fragment and the $5^{\prime} \mathrm{T}$ of the Hind III site at the $3^{\prime}$ end of the PCR fragment. The mutation $\mathrm{M} 2$ at position +36 of $S$. pombe $\mathrm{U} 2$ is also indicated. The three differences from the published RNA sequence (Brennwald et al.,1988) are as follows: nucleotide (nt) 84 , an additional $U$; nt 167 , only one $U$ instead of two; nt 187,3 ' end at $\mathrm{U}$. The predicted U2 RNA sequence is also shown; the end-points of the RNA coding region were determined by direct enzymatic RNA sequencing from the 3 ' end and by reverse transcriptase dideoxy-nucleotide sequencing of the RNA (Inoue and Cech, 1985) at the 5' end.
Fig. 2. Alignment of 5 ' flanking regions of snut, snu2, snu3 and snu4. The alignment of the TATA box regions of the SnRNA genes is shown together with the end-points of deletions $\Delta 4$ and $\Delta 5$. The start of the coding region is denoted by $\wedge \wedge \wedge$. Under the four sequences their consensus is given: lower-case letters show nucleotides common to the two sequences, and upper-case letters indicate that three out of four sequences match. Underlining indicates four out of four matches. 

G A T C
WM1 2345
CTAG
WM1 2345

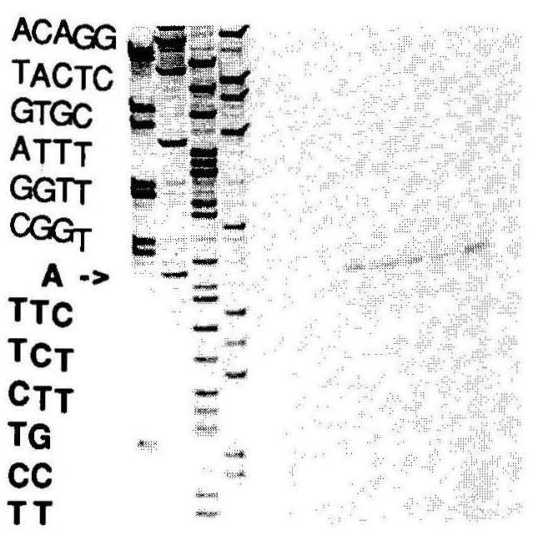

Fig. 3. Primer extension of snu2 deletion mutants. Left panel. Primer extension using a primer oligonucleotide specific for the M2 mutation present in the snu2 gene expressed from the promoter deletion constructs.

Right panel. Primer extension using a primer oligonucleotide capable of being extended on the mutant or wild-type U2. On the left margin the U2 gene sequence is given, the start of the RNA coding region is highlighted in bold, and the $5^{\prime}$ end of the U2 RNA is marked by an arrow. In both experiments DNA was sequenced in parallel using the same oligonucleotide as for the primer extension (lanes labelled GATC on the left panel and CTAG on the right, according to the dideoxy nucleotide used). The RNA used for each experiment was extracted from the wild type (lanes labelled W), or contained mutant RNA with undeleted upstream region (lanes labelled $M$ ) or having progressive deletions (lanes labelled 1 to 5 for the deletions $\Delta 1$ to $\Delta 5$ ). A darker exposure of the right panel is shown to allow the DNA sequence to be read. mutations $\Delta 2(-75)$ and $\Delta 3(-55)$ (see below) does not reduce transcription. Comparison of the alignments of snu2 with this region of snu4, and with the $5^{\prime}$ flanking regions of snu1 and snu3, did not reveal any regions in which the homology is shared by more than two snRNA genes, with the exception of the homology surrounding the TATA box (Fig. 2).

The snu2 gene is present as a single copy in the $S$. pombe genome (Dandekar and Tollervey, 1989) and gene-disruption experiments show it to be essential for viability (D. Tollervey, unpublished data). In order to follow the transcription of $\mathrm{U} 2$ from mutant promoter regions, it was therefore necessary to mark the coding region so as to allow it to be distinguished from the endogenous $\mathrm{U} 2$ gene. To do this, a single point mutation was introduced into the snu2 coding region (Fig. 1). Polymerase chain reaction $(P C R)$ was used to generate a series of fragments containing the mutant snu2 gene with end-points at various sites in the $5^{\prime}$ flanking region (Figs 1 and 2). These fragments were then cloned into the $S$. pombe replicating vector YEpU4 (Dandekar et al., 1989) and transformed into $S$. pombe. This plasmid carries the $2 \mu$ origin of replication; in $S$. pombe, such vectors replicate with low copy numbers ( $2-5$ copies per cell) (Beach et al., 1982).

To determine the level of expression of U2 from the mutant promoters, primer extension analyses were performed on total RNA using an oligonucleotide whose $3^{\prime}$ end corresponds to the site of the internal point mutation. This oligonucleotide therefore acts as a primer for reverse transcriptase on the mutant U2 (Fig. 3, left panel, lanes labelled M, 1, 2, 3, 4, and 5), but not the wild type (Fig. 3, left panel, lane W). Surprisingly, the level of synthesis of $\mathrm{U} 2$ obtained by the primer extension reaction in vitro from
RNA extracted from respective strains is similar to that from strains carrying plasmids containing $700 \mathrm{bp}$ of $5^{\circ}$ flanking sequence (Fig. 3 , left panel, lane $\mathrm{M}$ ) or the $\Delta 1$, $\Delta 2, \Delta 3$ or $\Delta 4$ mutations (Fig. 3 , left panel, lanes labelled $1-4$, respectively). In the $\Delta 4$ mutation, the $5^{\prime}$ flanking sequence of snu2 commences one nucleotide $5^{\prime}$ to the TATA box. This was confirmed by sequencing of the plasmid carrying snu2- $\Delta 4$, after recovery from the transformed strain of yeast. Deletion of the TATA box in $\Delta 5$ abolishes detectable transcription of snu2 (Fig. 3, left panel, lane 5). Primer extension using an oligonucleotide complementary to both the mutant and wild-type U2 (Fig. 3 , right panel) shows that the differences observed in the left panel are not due to variations in RNA recovery. The greater intensity of signal in the right panel lanes $M, 1,2$, 3 , and 4 is due to the presence of both the wild-type and M2 mutant U2 transcripts in these strains. The 5 ' ends of the U2 transcripts from $\Delta 1-\Delta 4$ are identical and correspond to the previously reported 5 ' end of U2 (Ares, 1986).

Northern hybridization using a short oligonucleotide probe complementary to the mutant $\mathrm{U} 2$ was also used to assess the expression from $\Delta 1-\Delta 4$ (Fig. 4) in vivo. This confirms the results shown in Fig. 3 . In addition, the hybridizing bands are identical in size, showing that the $3^{\prime}$ ends of the transcripts are also likely to be correct at the nucleotide level. In this experiment the expression from the undeleted plasmid (with $700 \mathrm{bp}$ of upstream sequence) is approximately two-fold higher than from the plasmid carrying the deletion mutations, indicating that sequences further upstream may also influence the level of transcription. However, up to deletion $\Delta 4$ there is still stable and efficient transcription of the U2 mutant RNA, which is abolished in deletion $\Delta 5$. The sequence around the TATA box, which is conserved between snu1, snu2, 
W M $\Delta 1 \quad \Delta 2 \quad \Delta 3 \quad \Delta 4 \quad \Delta 5$

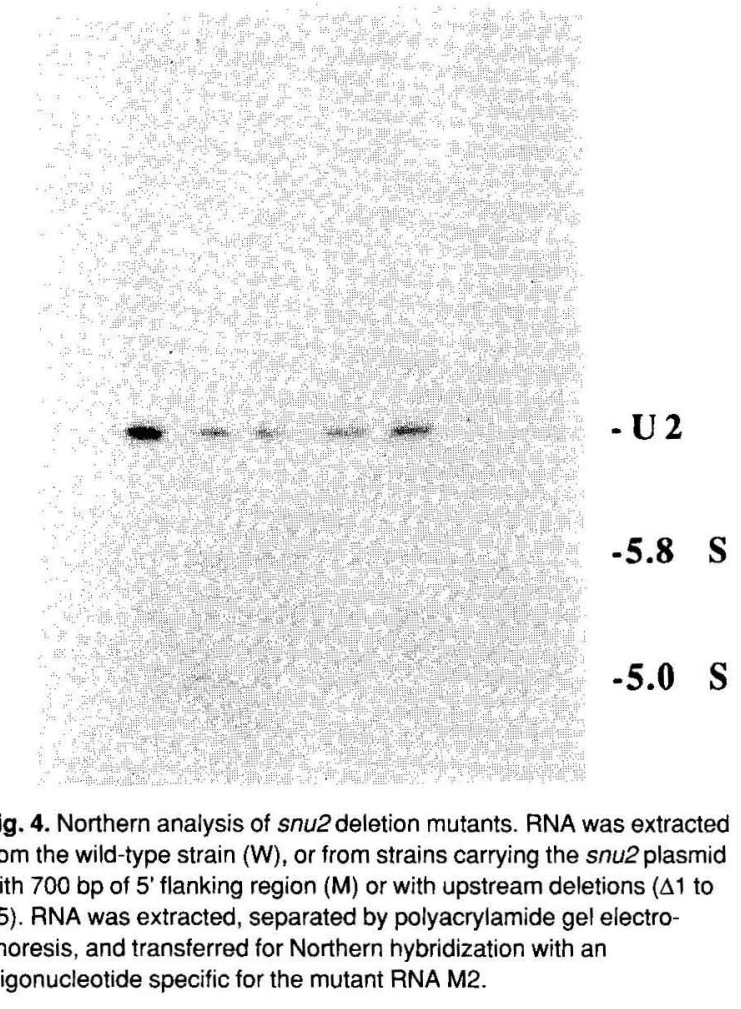

snu3 and snu4, is therefore necessary for efficient transcription of snu2. The region of 3 ' flanking sequence extending to +50 is also sufficient to direct accurate $3^{\prime}$ end formation.

We do not know whether the TATA region alone is sufficient to direct transcription, or whether additional sequences are required. These might lie between the TATA box and the transcription start site or within the coding sequence. Many genes transcribed by RNA polymerase III have promoter elements which lie within the coding sequence. However, we have detected no homology to such promoter elements in the snRNA genes described here. Moreover, U1, U2, U3, and U4 from $S$. pombe all have trimethyl guanosine 5'-cap structures (Dandekar and Tollervey, 1989), a feature typical of snRNAs transcribed by RNA polymerase II, rather than the $\gamma$-methyl phosphate found on the U6 snRNA (Singh and Reddy, 1989), which is transcribed by RNA polymerase III (Mattaj et al., 1988, reviewed by Parry et al., 1989). Some homology can be detected amongst the $S$. pombe snRNA genes in the region between the TATA box and the transcription start (Fig. 2), suggesting that this region might contain novel promoter elements. It also remains a formal possibility that sequences in the vector act in concert with the TATA box as activators of transcription.

The sequences required for accurate and efficient transcription of snu2 are shorter than those of many other eukaryotic genes transcribed by RNA polymerase II, including other snRNA genes (Tebb and Mattaj, 1988; reviewed by Parry et al., 1989; Dahlberg and Lund, 1988). However, efficient transcription from short promoter regions is known from other systems, e.g. for the vaccinia virus promoters (Davison and Moss, 1989). Moreover, some ( $10 \%$ of wild-type) transcription from a 44 -nucleotide promoter region is observed for U2 RNA transcription in the plant Arabidopsis thaliana (Vankan and Filipowicz, 1988).

It has been proposed (Dahlberg and Lund, 1988) that the specialized transcription signals of vertebrate $\mathrm{sn}$ RNAs are adaptations to the very high rate of SnRNA synthesis. However, the rate of transcription of $S$. pombe snRNA genes (2000 molecules per cell from one gene in 150 min doubling time, or about 1 snRNA per $4.5 \mathrm{~s}$ ) is not very different from that of HeLa cells ( 1 million molecules of $U 1$ per cell from 60 genes per diploid cell in $18 \mathrm{~h}$ doubling time, or about 1 snRNA per $4 \mathrm{~s}$ per gene). Thus, while the snRNA transcription signals of vertebrate UsnRNAs may well be adapted to allow high transcription rates, these can also be obtained from TATA-box-containing snRNA genes.

\section{Experimental procedures}

\section{Strains and media}

The growth and handling of $S$. pombe has been described previously (Gutz et al., 1974). The strain used for transformation and expression of snu2 carries $\mathrm{h}^{+}$, ura4- $\Delta 18$, leu1-32, and ade6-704, and was kindly provided by $\mathrm{Dr} \mathrm{J}$. Kohli.

\section{Mutant construction and plasmids}

To introduce mutation M2, a $1.5 \mathrm{~kb}$ genomic fragment containing the snu2 gene in bluescript (KS+) (Stratagene) was converted to the single-stranded form and mutagenized using an oligonucleotide (GGCTTAGATCAAGTGTACTATCTGTTCTTTTCAG) and a site-directed mutagenesis kit (Amersham, UK). This resulted in the change $\mathrm{G}^{19}-\mathrm{C}$ (the site of the mutation is shown in bold) in the snu2 coding region. Sequence analysis demonstrated that this was the only mutation in snu2. The $1.5 \mathrm{~kb}$ genomic fragment was subcloned into the $S$. pombe replicating plasmid, YEpU4 (Dandekar et al., 1989) to generate plasmid pM2, which contains $\approx 700$ bp of $5^{\circ}$ - and $600 \mathrm{bp}$ of $3^{\prime}$ flanking sequence. The $\Delta 1-\Delta 5$ constructs were generated using PCR with an oligonucleotide (AAAAGCTTCCATCCAATGTTGTCAAATTC) which introduces a Hindlll site (bold) at position +50 in the $3^{\prime}$ flanking region of snu2 and oligonucleotides which introduced EcoRl sites (bold) into the $5^{\circ}$

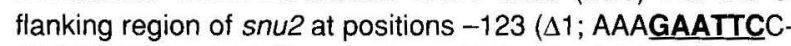
CTTCTGGTAACAAG), -73 ( $\triangle 2$; AAAGAATTCGCGAAGTAATCTCAGGGTTC), -53 ( $\triangle 3$; AAAGAATTCGGGTTATTTATGAGA), -33 ( $\triangle 4$; AAAGAATTCTATATATACAGGTACTCGTG), and -25 ( $\triangle 5$; AAAGAATTCAGGTACTCGTGCATTTGGTT). 
DNA was amplified in a Hybaid thermal cycler for 30 cycles of $15 \mathrm{~s}$ at $94^{\circ} \mathrm{C}, 30 \mathrm{~s}$ at $40^{\circ} \mathrm{C}$, and $1 \mathrm{~min}$ at $72^{\circ} \mathrm{C}$. The products were cleaved with ECORI and HindIII, gel-purified and cloned into YEpU4 (Dandekar et al., 1989). Plasmids were introduced into S. pombe using the Li-acetate method (Ito et al., 1983).

\section{RNA extraction and analysis}

RNA extraction and Northern hybridization were as previously described (Tollervey and Mattaj, 1987). The hybridization probe, specific for the M2 mutation, was of sequence CAGATAGTACAC. Primer extension was performed as previously - described (Inoue and Cech, 1985) using an oligonucleotide specific for the M2 mutant RNA (CTGAAAAGAACAGATAG) or - capable of being extended on either RNA (GAACAGATACTACACTTG).

\section{DNA sequencing}

DNA was sequenced by the dideoxy chain termination technique (Sanger et al., 1977) using a Sequenase kit (USB).

\section{Acknowledgement}

T.D. was supported by a grant from Boehringer Ingelheim Fonds for Basic Medical Research.

\section{References}

Ares, M. (1986) U2 RNA from yeast is unexpectedly large and contains homology to vertebrate U4, U5 and U6 small nuclear RNAs. Cell 47: 49-59.

Bally, M., Hughes, J., and Cesareni, G. (1988) snR30: a new, essential small nuclear RNA from Saccharomyces cerevisiae. Nucl Acids Res 16: 5291-5303.

Beach, D., Piper, M., and Nurse, P. (1982) Construction of a Schizosaccharomyces pombe gene bank in a yeast bacterial shuttle vector and its use to isolate genes by complementation. Mol Gen Genet 187: 326-329.

Brennwald, P., Porter, G., and Wise, J.A. (1988) U2 small nuclear RNA is remarkably conserved between Schizosaccharomyces pombe and Mammals. Mol Cell Biol 8: 55755580.

Carbon, P., Murgo, S., Ebel, J.P., Krol, A., Tebb, G., and Mattaj, I.W. (1987) A common octamer motif binding protein is involved in the transcription of U6 snRNA by RNA Polymerase III and U2 snRNA by RNA Polymerase II. Cell 51: 71-79.

Dahlberg, J.E., and Lund, E. (1988) The genes and transcription of the major small nuclear RNAs. In Structure and Function of Major and Minor Small Ribonucleoprotein Particles. Birnstiel, M.L. (ed.). Heidelberg: Springer-Verlag, pp. 3870.

Dandekar, T., and Tollervey, D. (1989) Cloning of Schizosaccharomyces pombe genes encoding the U1, U2, U3 and U4 snRNAs. Gene 81: 227-235.
Dandekar, T., Ribes, V., and Tollervey, D. (1989) Schizosaccharomyces pombe U4 small nuclear RNA closely resembles vertebrate $\cup 4$ and is required for growth. $J$ Mol Biol 208: 371-379.

Davison, A., and Moss, B. (1989) Structure of vaccinia virus late promoters. J. Mol. Biol. 210: 771-784.

Guthrie, C. (1988) Genetic analysis of yeast small nuclear RNAs. In Structure and Function of Major and Minor Small Ribonucleoprotein Particles. Birnstiel, M.L. (ed.). Heidelberg: Springer-Verlag, pp. 196-213.

Gutz, H., Heslot, H., Leupold, U., and Loprieno, N. (1974) Schizosaccharomyces pombe. In Handbook of Genetics. Volume 1. King, R.C. (ed.). New York: Plenum Press, pp. $395-446$.

Inoue, T., and Cech, T.R. (1985) Secondary structure of the circular form of the tetrahymena rRNA intervening sequence: a technique for RNA structure analysis using chemical probes and reverse transcriptase. Proc Natl Acad Sci USA 82: 648-652.

Ito, H., Fukuda, Y., Murata, K., and Kimura, A. (1983) Transformation of intact yeast cells treated with alkali cations. J Bacteriol 153: 163-168.

Maniatis, T., and Reed, R. (1987) The role of small nuclear ribonucleoprotein particles in pre-mRNA splicing. Nature 325: $673-678$.

Mattaj, I.W., Dathan, N.A., Parry, H.D., Carbon, P., and Krol, A. (1988) Changing the RNA polymerase specificity of $U$ snRNA gene promoters. Cell, 55: 435-442.

Parry, H.D., Scherly, D., and Mattaj, I.W. (1989) 'Snurpogenesis': the transcription and assembly of UsnRNP components. Trends Biochem Sci, 14: 15-19.

Porter, G.L., Brennwald, P.J., Holm, K.A., and Wise, J.A. (1988) The sequence of U3 from Schizosaccharomyces pombe suggests structural divergence of this snRNA between metazoans and unicellular eukaryotes. Nucl Acids Res 16: 10131-10152.

Porter, G.L., Brennwald, P., and Wise, J.A. (1990) U1 small nuclear RNA from Schizosacchaaromyces pombe has unique and conserved features and is encoded by an essential single copy gene. Mol Cel Biol 10: 2874-2881.

Russell, P. (1983) Evolutionary divergence of the mRNA transcription initiation mechanism in yeast. Nature 301: 167-169.

Sanger, F., Nicklen, S., and Coulson, A.R. (1977) DNA sequencing with chain terminating inhibitors. Proc Natl Acad Sci USA 74: 5463-5467.

Singh, R., and Reddy, R. (1989) $\gamma$-monomethyl phosphate: A cap structure in spliceosomal U6 small nuclear RNA. Proc Natl Acad Sci USA 86: 8280-8283.

Tebb, G., and Mattaj, I.W. (1988) Positionally exact initiation is required for the formation of a stable RNA polymerase II transcription complex in vivo. EMBO J7: 3785-3792.

Tollervey, D., and Mattaj, I.W. (1987). Fungal small nuclear ribonucleoproteins share properties with plant and vertebrate U-snRNPs. EMBO J 6: 469-476.

Vankan, P., and Filipowicz, W. (1988) Structure of U2 snRNA genes of Arabidopsis thaliana and their expression in electroporated plant protoplasts. EMBO J 7: 791-799.

Woolford, J.L. (1989) Nuclear pre-mRNA splicing in yeast. Yeast 5: 439-457. 